\title{
A Note on Maximality of Ideal-independent Sets
}

\author{
Corey T. Bruns \\ University of Wisconsin-Whitewater \\ Email: brunsc@uww.edu
}

\section{INTRODUCTION AND DEFINITIONS}

$\mathrm{W}$

e give a solution to a problem originally posed in a draft of J.D. Monk

[4].This result is now listed as further fact 4 after theorem 2.16. In doing so, we will consider some subsets of boolean algebras.

We will follow $\mathrm{S}$. Koppelberg [2] for notation. In particular,$+ \cdot$, and - will used as the Boolean operators, and 0 and 1 as the least and greatest element. By extension, the least upper bound of a set $M$ will be denoted $\sum M$

Definition 1.1. A subset $X$ of a boolean algebra is ideal-independent if $0,1 \notin X$ and $\forall x \in X, x \notin\langle x \backslash\{x\}\rangle^{i d}$; equivalently, for distinct $x, x_{1}, \ldots, x_{n} \in X$, $x \not x_{1}+x_{2}+\ldots+x_{n}$.

By Zorn's lemma, there are maximal ideal-independent sets.

This is used in one of several equivalent definitions in Monk [3] of the spread $s$ of a boolean algebra $A$ :

$$
s(A)=\sup \{|X|: X \text { is ideal-independent. }\}
$$

Related to this cardinal function is the minimaximal version:

$$
s_{m m}(A) \stackrel{\text { def }}{=} \min \{|X|: X \text { is infinite and maximal ideal-independent }\} .
$$

Among the results of Monk [4] is that it is consistent with ZFC that

$$
\aleph_{0}<s_{m m}(\mathscr{P}(\omega) / \text { fin })<\beth_{1} \text {. }
$$

Mathematical Journal of Interdisciplinary Sciences

Vol. 1, No. 1, July 2012 pp. $83-86$

\section{MAXIMALITY}

Our main result is a necessary condition for maximality of an ideal-independent set.

Theorem 2.1. Let $A$ be a boolean algebra.If $X \subseteq A$ is maximal for idealindependence, then $\sum X=1$.

CHITKARA 司 UNIVERSITY

C 2012 by Chitkara University. All Rights Reserved. 
Proof. Let $X \subseteq A$ be ideal-independent and let $b \in A \backslash\{1\}$ be such that $\forall x \in X, x \leq b$. We claim that $X^{\prime \text { def }}=X \bigcup\{-b\}$ is ideal-independent (thus proving the theorem).

We need to show that for distinct $x, x_{1}, x_{2}, \ldots, x_{n} \in X^{\prime}, x \neq x_{1}+x_{2}+\ldots+x_{n}$. There are three cases to consider.

1. $-b \notin\left\{x, x_{1}, x_{2}, \ldots, x_{n}\right\}$.

Then $x \not x_{1}+x_{2}+\ldots+x_{n}$ by the ideal-independence of $X$.

2. $-b=x$.

Assume otherwise, that is, $-b \leq x_{1}+x_{2}+\ldots+x_{n}$. Since $x_{1}+x_{2}+\ldots$ $+x_{n} \leq b$, we have $-b \leq b$, thus $b=1$, contradiction.

3. $-b=x_{1}$

Then as $x \in X, x \leq b$, that is, $x \cdot-b=0$. By the ideal-independence of $X$, $x \not \neq x_{2}+x_{3}+\ldots+x_{n}$, so that $y_{\text {def }}^{=} \cdot x\left(x_{2}+x_{3}+\ldots+x_{n}\right)<x$.

Then

$$
x \cdot\left(-b+x_{2}+x_{3}+\ldots+x_{n}\right)=x \cdot-b+x \cdot\left(x_{2}+x_{3}+\ldots+x_{n}\right)=0+y<x,
$$

thus $x \not \neq-b+x_{2}+x_{3}+\ldots+x_{n}$.

It is worth noting that a converse of Theorem 2.1 does not hold for infinite sets. That is, there is an ideal-independent set $X$ with $\sum X=1$ that is not maximal.

Proposition 2.2. Let $\mathbb{P}=\{p \in \omega: p$ is prime $\}$. In the boolean algebra $\mathscr{P}(\omega$ $\backslash\{0,1\})$ where + is union, $\cdot$ is intersection, and $-x$ is $(\omega \backslash\{0,1\}) \backslash x$, set $X=$ $:\left\{p \mathbb{Z}^{+}: p \in \mathbb{P}^{\}}\right.$(where $n \mathbb{Z}^{+}$is the set of all nonzero multiples of $n$ ). Then all of the following are true:

1. $\sum X=1$

2. $X$ is an ideal-independent set.

3. $X \cup\{\mathbb{P}\}$ is also ideal-independent.

\section{Proof.}

1. $\bigcup X=\omega \backslash\{0,1\}$ as every integer other than 0 and 1 is a nonzero multiple of a prime, so $\sum X=1$

2. If $p$ is prime, then $p$ is not the multiple of any other prime, so $p \mathbb{Z}^{+} \not \neq p_{1} \mathbb{Z}^{+} p_{2} \mathbb{Z}^{+}+\ldots+p_{n} \mathbb{Z}^{+}$if $p, p_{1} \ldots, p_{\mathrm{n}}$ are all distinct primes.

Thus $X$ is ideal-independent. 
3. We have two parts to show that $X \cup\{\mathbb{P}\}$ is ideal-independent; Let $Y$ be a finite subset of $X$ and $q, r$ distinct primes such that $q \mathbb{Z}^{+} \notin Y$ and $r \mathbb{Z}^{+} \notin Y$. We must show that $\mathbb{P} \not \sum Y$ and that. $q \mathbb{Z}^{+} \not \mathbb{P}+\sum Y$.

$q \in \mathbb{P}$ and $q \notin \sum Y$, so the first is true.

$r q \in q \mathbb{Z}^{+}$, but $r q \notin \mathbb{P}$ as it is composite and $r q \notin \sum Y$ since neither $r \mathbb{Z}^{+} \in Y$ nor $\mathrm{q} \mathbb{Z}^{+} \in Y$.

Thus $X \cup\{\mathbb{P}\}$ is ideal-independent and so $X$ is not maximal.

Corollary 2.3. For $A$ infinite, $\mathfrak{p}(A) \leq s_{m m}(A)$; in particular $\mathfrak{p}=\mathfrak{p}(\mathscr{P}(\omega) /$ fin $) \leq s_{m m} \mathscr{P}(\omega) /$ fin $)$.

We recall from Monk [3] that the pseudo-intersection number $\mathfrak{p}$ is defined as:

$$
\mathfrak{p}(A) \stackrel{\text { def }}{=} \min \left\{|Y|: \sum Y=1 \text { and } \sum Y^{\prime} \neq 1 \text { for every finite } Y^{\prime} \subseteq Y\right\} .
$$

Proof. Let $X$ be maximal ideal-independent (and thus infinite). By Theorem 2.1, $\sum X=1$

If $X^{\prime} \subseteq X$ is finite, $\sum X^{\prime} \neq 1$, otherwise, take $x \in X \backslash X^{\prime}$, then $x \leq 1=\sum X^{\prime}$, contradicting the ideal-independence of Thus

and so $\mathfrak{p}(A) \leq s_{m m}(A)$

$$
\{|X|: X \text { is maximal ideal-independent }\} \subseteq
$$

$$
\left\{|Y|: \sum Y=1 \text { and } \sum Y^{\prime} \neq 1 \text { for every finite } Y^{\prime} \subseteq Y\right\}
$$

Now, $\mathfrak{p}$ is one of the smaller continuum cardinals, so that $s_{m m}$ is larger is not particularly surprising. However, the method of proof does turn out to be useful with other similar properties.Other set properties involving sums of finite subsets, such as independence and $\mathrm{n}$-independence, are amenable to these methods.In [1], we introduce some variations on $\mathrm{i}$; their placement in order of continuum cardinals can be similarly determined.

\section{REFERENCES}

[1] Bruns, C.T.: A simultaneous generalization of independence and disjointness in Boolean algebras.Order pp. 1-21. DOI: 10.1007/s11083-011-9237-x

[2] Koppelberg, S.: General theory of Boolean algebras, pp. xix + 3121. North-Holland, Amsterdam, The Netherlands (1989)

[3] Monk, J.D.: Cardinal invariants on Boolean algebras, Progress in Mathematics, vol. 142. Birkhäuser Verlag, Basel (1996). http://dx.doi.org/10.1007/978-3-0346-0334-8

[4] Monk, J.D.: Maximal irredundance and maximal idealindependence in Boolean algebras. J. Symbolic Logic 73(1), 261-275(2008). URL http://projecteuclid.org/getRecord?id=euclid. jsl/ 1208358753. http://dx.doi.org/10.2178/jsl/1208358753

Corey T. Bruns is a lecturer in mathematics at the University of Wisconsin-Whitewater. 
